\title{
Modified-release Soft Capsule Form
}

National Cancer Institute

\section{Source}

National Cancer Institute. Modified-release Soft Capsule Form. NCI Thesaurus. Code C149664.

Solid single-dose preparation consisting of a soft shell containing a semi-solid or liquid formulation, showing a rate, a place and/or a time of release different from that of a conventional-release capsule. This deliberate modification is achieved by a special formulation design and/or manufacturing method. 